\title{
Análise Numérica Preliminar do Escoamento em uma Coluna de Bolhas Descendente
}

\author{
Melo, L.D.V. ${ }^{1}$; Franco, V.P. ${ }^{4}$; Pinheiro, V.P. ${ }^{1,2,3 *}$; Martins, R.S. ${ }^{1}$ \\ 1 PRPPGE/UVV, Programa de Iniciação Científica em Métodos Numéricos, Universidade Vila Velha, \\ Vila Velha, ES, Brasil. \\ 2 PPGEM/UFES -Programa de Pós-Graduação em Engenharia Mecânica, Universidade Federal do \\ Espírito Santo, Vitória, ES, Brasil. \\ 3 CCTM/IFES - Coordenadoria do Curso Técnico em Mecânica, Instituto Federal do Espírito Santo, \\ Campus Guarapari, ES, Brasil. \\ 4 IGFE/ArcelorMittal Tubarão - Gerência de Área de Manutenção dos Altos Fornos 2 e 3, Serra, ES, \\ Brasil.
}

* e-mail: vitor.pinheiro1987@gmail.com

\section{Resumo}

No contexto de pesquisas correlatas aos escoamentos multifásicos turbulentos, os relatos experimentais e numéricos disponíveis na literatura sobre coluna de bolhas descendente ainda são relativamente escassos. Avanços substanciais em termos de modelos multifásicos, modelos de turbulência e novas propostas constitutivas para forças interfaciais comprovam que a linha de pesquisa é ativa e demanda investigação científica mais intensa. Nesta linha, o vigente trabalho visa efetuar, de forma preliminar, uma análise numérica de um escoamento descendente em padrão de bolhas, num duto cilíndrico vertical com a utilização do pacote comercial de dinâmica dos fluidos computacional ANSYS-Fluent. A geometria utilizada na experimentação numérica apresenta um tubo vertical com diâmetro interno de $20 \mathrm{~mm}$, a fase dispersa é caracterizada por diâmetro médio de bolha de $2,2 \mathrm{~mm}$ e com fração volumétrica de injeção de gás de $3 \%$. A validação dos resultados é feita através comparação das curvas numéricas com resultados experimentais bem estabelecidos na literatura. A nível de análise, o parâmetro de velocidade superficial de injeção do líquido é apreciado com o objetivo de estabelecer sua influência sobre os perfis de velocidade axial da fase contínua e sobre a distribuição da fração de vazios na direção radial.

Palavras-Chave: Escoamento Multifásico; Coluna de Bolhas; Escoamento Descendente; Perfil de Velocidade; Zona Livre de Bolhas.

\section{Introdução}

O estudo da dinâmica dos escoamentos multifásicos tem relevância crescente em diversas áreas da indústria tais como nos setores de óleo e gás, siderurgia, alimentício bem como papel e celulose. $\mathrm{O}$ desafio correlato ao tema reside na dificuldade de entender adequadamente a fenomenologia física, tal como gerar uma modelagem matemática adequada a cada tipo de situação. Os relatos experimentais e numéricos disponíveis na literatura sobre coluna de bolhas descendente ainda são relativamente escassos se comparados com escoamentos ascendentes.

O escoamento bifásico descendente constitui linha de pesquisa muito ativa, na qual diversos pesquisadores se destacam, entre eles elencam-se os trabalhos de Ganchev et al.[1] e Kashinsky et al. [2], [6], que relatam de forma consolidada a existência de uma região livre de bolhas próxima a parede, que permite uma maior aceleração do líquido a depender das condições do escoamento. 


\section{Blucher Proceedings XI Encontro Científico de Física Aplicada

$\mathrm{O}$ vigente projeto delimita-se a um estudo preliminar do escoamento em uma coluna de bolhas água-ar descendente em um duto cilíndrico. A principal diretriz deste artigo é realizar uma análise paramétrica da velocidade axial e da fração de vazios em relação a velocidade de injeção da fase contínua. As simulações numéricas são performadas com auxílio da ferramenta computacional ANSYS Fluent e validadas com dados experimentais de [2].

\section{Modelagem Matemática}

A descrição hidrodinâmica de um escoamento multifásico e suas interações correlatas pode ser feita através de algumas abordagens, dentre as quais se destacam o modelo de mistura, euleriano e volume de fluido [5]. Neste projeto utiliza-se o modelo euleriano, que tem como premissa básica um campo de velocidade individual para cada fase, com isso cada fase terá sua equação de momento. As equações de governo hidrodinâmico são representadas respectivamente pelas equações 1 e 2 .

$$
\begin{gathered}
\frac{\partial}{\partial t}\left(\alpha_{q} \rho_{q} \overrightarrow{u_{q}}\right)+\nabla \cdot\left(\alpha_{q} \rho_{q} \vec{u}_{q} \overrightarrow{u_{q}}\right)= \\
-\alpha_{q} \nabla P+\alpha_{q} \rho_{q} \vec{g}+\nabla \cdot \overline{\bar{\tau}_{q}}+ \\
\sum_{p=1}^{n}\left(\overrightarrow{R_{p q}}+\dot{m}_{p q} \overrightarrow{u_{p q}}-\dot{m}_{q p} \overrightarrow{u_{q p}}\right)+ \\
\left(\vec{F}_{q}+\vec{F}_{l, q}+\vec{F}_{w l, q}+\vec{F}_{v m, q}+\vec{F}_{t d, q}\right) \\
\frac{\partial}{\partial t}\left(\alpha_{q} \rho_{q}\right)+\nabla \cdot\left(\alpha_{q} \rho_{q} \vec{v}_{q}\right)= \\
\sum_{p=1}^{n}\left(\dot{m}_{p q}-\dot{m}_{q p}\right)+S_{q}
\end{gathered}
$$

Os últimos termos que são descritos na equação 1 , refere-se as interações interfaciais entre as fases, sendo elas, força de arrasto, força de massa virtual, força de parede, sustentação, dispersão turbulenta, dentre outras que devem ser modeladas de acordo com as condições de contorno do problema.
O tratamento da turbulência é abordado via modelos RANS devido a sua simplicidade e eficiência. Nesta categoria, dentre os modelos disponíveis, seleciona-se o SST $\kappa-\omega$, devido a sua boa performance em problemas de natureza correlata, provavelmente ligada a uma boa capacidade de descrever as zonas perto da parede [3].

$\rho \overline{u_{j}} \frac{\partial k}{\partial x_{j}}=\frac{\partial}{\partial x_{i}}\left[\left(\mu+\frac{\mu_{T}}{\sigma_{\varepsilon}}\right) \frac{\partial k}{\partial x_{i}}\right] \varepsilon+\rho P_{k}-\rho$

$$
\begin{gathered}
\frac{\partial(\rho \omega)}{\partial t}+\nabla \cdot(\rho \omega U)=\nabla \cdot\left[\left(\mu+\frac{\mu_{\tau}}{\sigma_{\omega, 1}}\right) \nabla \omega\right] \\
-\beta \rho \omega^{2}+\gamma_{2}\left(2 \rho S_{i j} \cdot S_{i j}-\frac{2}{3} \rho \omega \frac{\partial U_{i}}{\partial x_{j}} \delta_{i j}\right)+ \\
+2 \frac{\rho}{\sigma_{\omega, 2} \omega} \frac{\partial \kappa}{\partial x_{\kappa}} \frac{\partial \omega}{\partial x_{\kappa}}
\end{gathered}
$$

As equações 3 e 4 compõem o modelo, onde, $\kappa$ representa a energia cinética turbulenta gerada e $\omega$ contabiliza a taxa de dissipação específica. O ajuste fino do modelo de turbulência em questão se dá pela calibração do valor das constantes $\sigma_{\kappa}, \sigma_{\omega, 1}$, $\sigma_{\omega, 2}, \gamma_{2}, \beta_{2}$ e $\beta *$ segundo a literatura correlata [3].

\section{Metodologia Adotada}

$\mathrm{Na}$ entrada do domínio, impõe-se um perfil de velocidade uniforme para as duas fases do escoamento, com uma distribuição de fração volumétrica de gás de $3 \%$ e diâmetro de bolha de 2,2mm. A saída do domínio é descrita por uma condição de pressão atmosférica e um modelo de fluxo reverso, que utiliza-se dos parâmetros pertencentes as células vizinhas. A condição de não deslizamento na parede é aplicada. A natureza axissimétrica da tubulação permite a proposta de uma simulação bidimensional baseada num retângulo de revolução. A geometria possui um diâmetro $D=20 \mathrm{~mm}$ e um comprimento de $100 D$, devidamente representados na Figura 1. Todos os resultados analisados na sequência são retirados na posição axial $y / D=100$. 


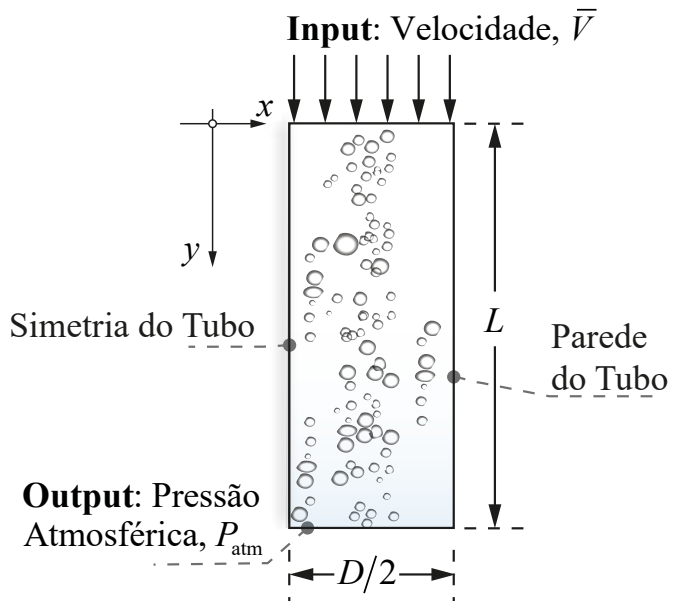

Figura 1: Condições de Contorno.

As principais forças para determinar o posicionamento das bolhas em escoamentos descendentes são ilustradas na Figura 2.

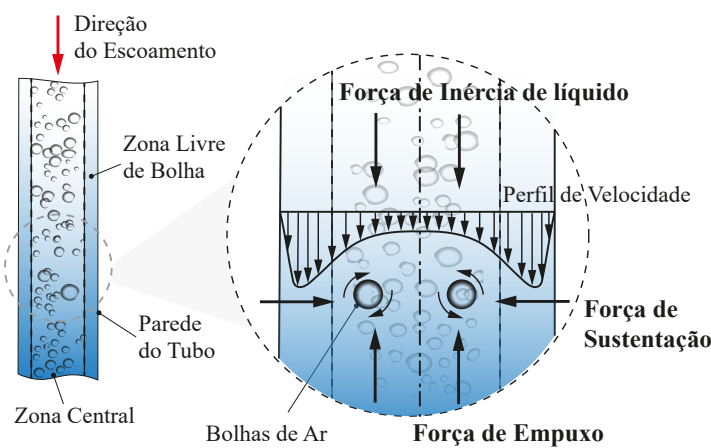

Figura 2: Balanço de Forças.

Os modelos constitutivos utilizados para descrever as forças interfaciais são: Tomiyama com modificação de Brucato para o arrasto, Tomiyama para sustentação, Lopez e Bertodano para dispersão turbulenta, Hosokawa com proposta de Frank para a força de parede, modelo CSF para tensão superficial e a massa virtual foi negligenciada por não provocar alteração significante no escoamento [4].

Uma importante etapa preliminar para a geração de resultados consiste na realização de um teste de malha com refinamento progressivo, a fim de determinar uma malha que não mais interfira nos resultados do problema. Neste trabalho foram testadas quatro malhas cujos dados e características são mostrados na Tabela 1.

\begin{tabular}{c|c|c} 
Malha & Elementos & $y^{+}$ \\
\hline 1 & 40000 & 12,1 \\
\hline 2 & 60000 & 8,31 \\
\hline 3 & 80000 & 6,16 \\
\hline 4 & 100000 & 4,91
\end{tabular}

Tabela 1: Descrição das Malhas.
A visualização gráfica dos resultados do teste de malha é mostrada pela Figura 3.

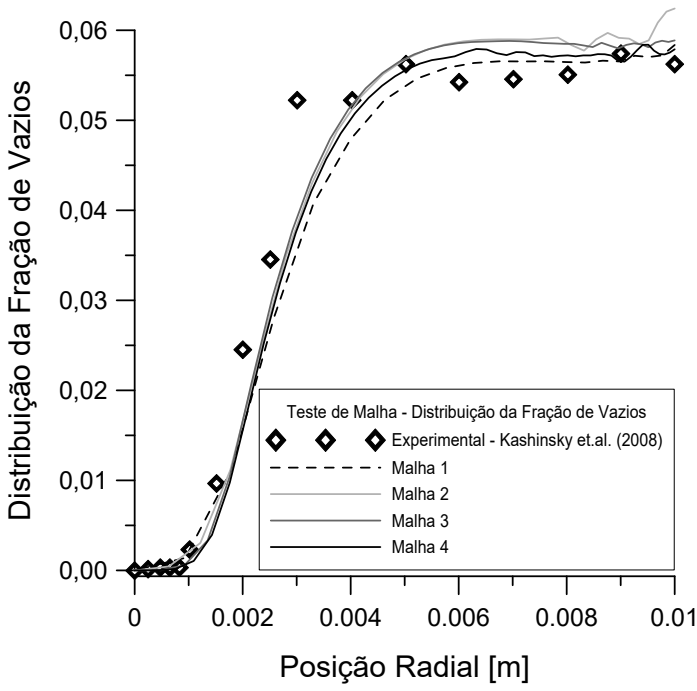

Figura 3: Teste de Malha.

Os valores da distância adimensional em relação a parede, $y^{+}$, em conjunto com o comportamento das curvas de malha apontam a malha 4 como a mais adequada para performar as simulações a porvir.

\section{Análise de Resultados}

No intuito de comparar o efeito da intensidade da força de inércia do escoamento em relação ao empuxo sofrido pela bolha, exposto na Figura 2, varia-se a magnitude da velocidade da fase contínua na entrada do domínio computacional, como pode ser visualizado na Figura 4.

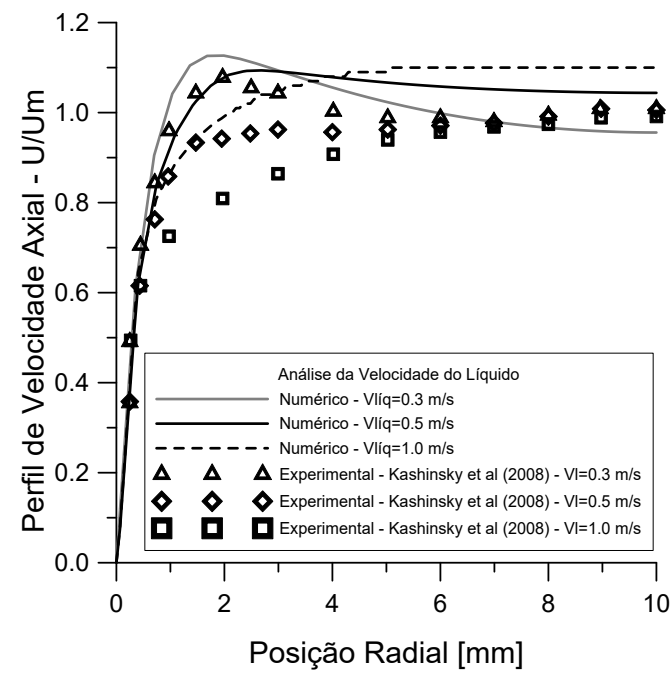

Figura 4: Perfil de Velocidade Axial do Líquido.

A tendência física esperada com o aumento gradual da velocidade do líquido traduz-se numa zona livre de bolhas cada vez 


\section{Blucher Proceedings XI Encontro Científico de Física Aplicada

mais delgada, que por sua vez tende a desacelerar o escoamento na zona próxima a parede, o que ameniza a presença dos picos de velocidade. Este efeito pode ser explicado de forma adequada pelo aumento da ordem de grandeza das forças de inércia frente as forças de empuxo [6].

A aderência entre a simulação numérica e os resultados experimentais de Kashinsky et al. [2], foi satisfatória para as velocidades mais baixas e descola-se mais dos resultados experimentais a medida que essa intensidade aumenta. Apesar disso, o comportamento qualitativo dos picos de velocidade, foi bem descrito nos resultados numéricos.

O segundo resultado nessa análise diz respeito à distribuição da fração de vazios na direção radial ao escoamento como pode ser visto na Figura 5.

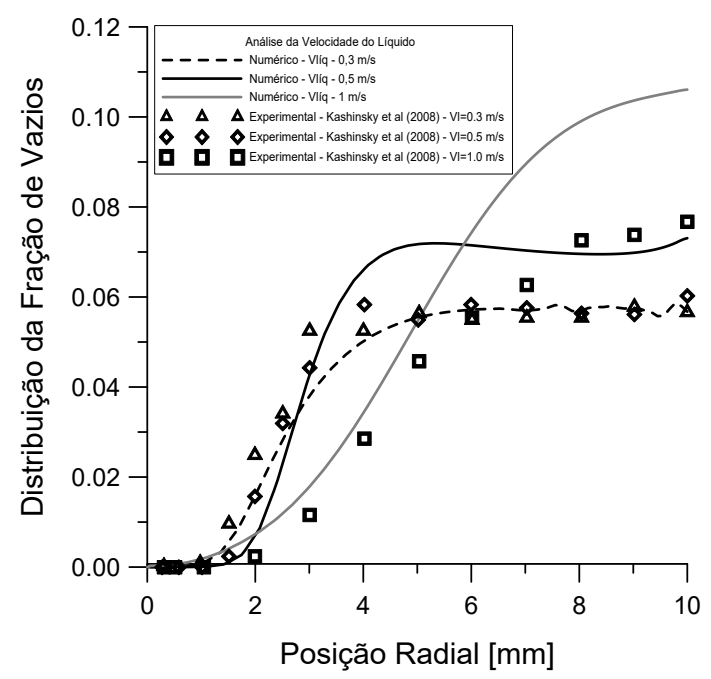

Figura 5: Fração de Vazios na direção radial.

As curvas numéricas confirmam a tendência física de uma melhor distribuição das bolhas ao longo da direção radial a medida que as forças inerciais ganham importância relativa. Houve uma boa concordância dos resultados numéricos gerados e dos resultados experimentais, novamente com destaque para as curvas cujos efeitos inerciais são menos intensos. Na Figura 5 faz-se notável uma região próxima a parede que apresenta fração volumétrica nula, região esta que conceitua a zona livre de bolhas de um escoamento descendente [6].

\section{Considerações Finais}

As simulações performadas apresentaram moderada concordância quando comparadas aos dados experimentais para baixas frações volumétricas de gás. Os resultados preliminares foram satisfatórios e ensejam um estudo de malha mais sistêmico e a testagem de outras configurações de modelos de forças interfaciais, de modo a conferir um maior nível de aderência quantitativa, e por consequência permitir a extrapolação das simulações numéricas para cenários de frações volumétricas de gás mais elevadas.

\section{Referências}

[1] Ganchev, B.G., and PeresadKo, V.G. Processes of hydrodynamics and heat exchange in descending bubble flows. Journal of engineering physics 49.2 (1986): 879-886.

[2] Kashinsky, O.N. and Lobanov, P.D. and Randin, V.V. The influence of a small gas addition to the structure of gasliquid downward flow in a tube, Springer, 2008, vol. 17, pp. 120.

[3] Menter, F.R. Performance of popular turbulence model for attached and separated adverse pressure gradient flows, AIAA, 1992, vol. 30, pp. 2066-2072.

[4] Fluent, ANSYS. ANSYS Fluent theory guide: Version 15.0, Ansys Inc., Canonsburg, 2013.

[5] Rosa, E.S. Escoamento multifásico isotérmico: modelos de multifluidos e de mistura, Bookman Editora, 2009.

[6] Kashinsky, O.N. et al. Experimental and numerical study of downward bubbly flow in a pipe. International journal of heat and mass transfer, v. 49, n. 19-20, p. 3717-3727, 2006. 